\title{
Inteligencia emocional del docente y satisfacción académica del estudiante universitario
}

\author{
Daniel Rubén Tacca Huamán* \\ Universidad Tecnológica del Perú, Lima, Perú. \\ https://orcid.org/0000-0002-0694-5262 \\ Ana Luisa Tacca Huamán** \\ Asociación Educativa Estándares, Lima, Perú. \\ https://orcid.org/0000-0003-2068-7527 \\ Renzo Cuarez Cordero*** \\ Universidad Nacional Mayor de San Marcos, Lima, Perú. \\ https://orcid.org/0000-0003-4173-2876
}

Recibido: 26/02/2019 Revisado: 23/07/2019 Publicado: 14/04/2020

\begin{abstract}
Resumen
En el siglo XXI, el docente universitario se ha convertido en un agente mediador entre el estudiante y el conocimiento; además, las habilidades personales y profesionales del docente participan activamente en el aprendizaje del estudiante. El propósito de la investigación fue conocer la relación entre la inteligencia emocional del docente (modelo de Bar-On) y la satisfacción académica del estudiante universitario, vista como parte del bienestar psicológico (posición eudaimónica). La investigación fue correlacional. Se evaluó la inteligencia emocional de 87 docentes y se recogió información sobre la satisfacción académica de 597 estudiantes. Se encontró que existe correlación positiva entre las variables de estudio (.80), siendo el componente interpersonal el que obtuvo mayor coeficiente de correlación con la satisfacción académica. Además, se observó que el 91\% de los docentes obtuvo niveles promedio y alto de inteligencia emocional; las docentes mujeres presentaron mayor puntaje en esta variable que los varones, aunque las diferencias no fueron significativas. Adicionalmente, los docentes de 45 años a más mostraron mayor desarrollo de habilidades emocionales y mayor satisfacción académica por parte de los estudiantes.
\end{abstract}

Palabras clave: Emoción; satisfacción; enseñanza superior; profesor de universidad; estudiante universitario

\section{Emotional intelligence of the teacher and academic satisfaction of the university student}

\begin{abstract}
In the XXI century, the university professor has become a mediating agent between the student and knowledge; in addition, the personal and professional skills of university professors are actively involved in student learning. The purpose of the research was to know the relation between the emotional intelligence of university professors (Bar-On model) and the academic satisfaction of university students, seen as part of the psychological well-being (eudaimonic position). The research was correlational and transversal, emotional intelligence was assessed to 87 professors and information on academic satisfaction was collected in 597 students. The results indicated that the study variables correlate positively (.80), being the interpersonal component to have the highest correlation coefficient with the academic satisfaction. Further-
\end{abstract}


more, it was observed that $91 \%$ of professors obtained average and high levels of emotional intelligence; women professors obtained higher scores than men professors, but the differences were not significant. Additionally, professors over 45 years of age showed greater development of emotional skills and greater academic satisfaction from the students.

Keywords: Emotion; satisfaction; higher education; university professor; university student

\title{
Inteligência emocional do professor e satisfação acadêmica do estudante universitário
}

\begin{abstract}
Resumo
No século XXI, o professor universitário tornou-se um mediador entre o aluno e o conhecimento; Além disso, as habilidades pessoais e profissionais dos professores universitários estão ativamente envolvidas na aprendizagem dos alunos. O objetivo da investigação foi conhecer a relação entre a inteligência emocional de professores universitários (modelo Bar-On) e a satisfação acadêmica de universitários, vistos como parte do bem-estar psicológico (posição eudaimônica). A investigação foi correlacional e transversal, a inteligência emocional foi avaliada em 87 professores e informações sobre a satisfação acadêmica foram coletadas em 597 alunos. Os resultados indicaram que as variáveis do estudo se correlacionam positivamente (0,80), sendo o componente interpessoal o de maior coeficiente de correlação com a satisfação acadêmica. Além disso, observou-se que 91\% dos professores obtiveram níveis médios e altos de inteligência emocional; as professoras obtiveram escores mais altos que os professores homens, más as diferenças não foram significativas. Além disso, professores com mais de 45 anos apresentaram maior desenvolvimento de habilidades emocionais e maior satisfação acadêmica por parte dos estudantes.
\end{abstract}

Palavras chave: Emoção; satisfação; ensino superior; professor; universidade estudante

Como citar:

Tacca, D., Tacca, A., \& Cuarez, R. (2020). Inteligencia emocional del docente y satisfacción académica del estudiante universitario. Revista Digital de Investigación en Docencia Universitaria, 14(1), e1085. https://doi.org/10.19083/ ridu. 2020.887

E n el paradigma tradicional de la enseñanza, el docente era considerado como dueño del conocimiento, pero la globalización y el ingreso al nuevo milenio hicieron posible una reconfiguración en la dinámica educativa: el estudiante se convirtió en protagonista y centro del proceso de enseñanza-aprendizaje en todos los niveles educativos. El docente universitario del siglo XXI debe dominar temáticas específicas propias de su área, conocer el contexto cultural de la asignatura, manejar estrategias didácticas, programación y tipos e instrumentos de evaluación; y el rol que cumple debe estar enfocado en el aprendizaje y no tanto en la enseñanza (Monereo \& Pozo, 2003). También, debe dejar de lado el "dictar una clase" y pasar a generar aprendizajes significativos y construir ambientes emocionalmente seguros y adecuados para desarrollar habilidades en los es- tudiantes. El docente debe realizar todo esto sin descuidar las tareas de investigación.

El éxito del docente universitario no solo responde al dominio de contenidos y estrategias, sino también a la capacidad de adaptarse y tomar en consideración las necesidades de los estudiantes (Almiron \& Porro, 2014). Las habilidades cognitivas constituyen un factor importante en la formación universitaria, pero también no hay que soslayar el rol trascendental de las emociones y los sentimientos (García-Rangel, García, \& Reyes, 2014). Por su parte, Golombek y Doran (2014), en una investigación sobre la formación docente, evidenciaron que las emociones se vinculan con los resultados de la enseñanza; otro estudio encontró que los futuros docentes reconocen la importancia de las emociones, argumentando que estas inciden en el aprendizaje, en el ambiente y en las metodologías em- 
pleadas (Buitrago, Avila, \& Cárdenas, 2017).

La efectividad y calidad del proceso de enseñanza tienen relación con las competencias sociales y emocionales del docente (Pertegal-Felices, Castejón-Costa, \& Martínez, 2011). Las competencias socioafectivas que se espera observar en un docente son conocimiento de sí mismo, autorregulación emocional, expresión de emociones, autoestima, empatía, entre otras (Fernández, Palomero, \& Teruel, 2009). Por ejemplo, Carcausto (2016) y Contreras y Dextre (2016), encontraron que los docentes exponen niveles promedios en el desarrollo de habilidades emocionales. Por otro lado, se ha demostrado que los aspectos emocionales de los docentes comprometen su desempeño diario y el aprendizaje de los estudiantes (Jennings \& Greenberg, 2009; Thomson \& Palermo, 2014). Altos niveles en el desarrollo de habilidades emocionales permiten crear ambientes adecuados para un efectivo aprendizaje (Palomera, Briones, \& Gómez-Linares, 2017). El manejo de las emociones en los docentes influye en su salud física y mental, así como en su desempeño laboral (Peñalva, López, \& Landa, 2012).

La inteligencia emocional era considerada como un aspecto poco relevante en la vida social hasta finales de la década de 1980 (Maidana \& Samudio, 2018), pero gracias a los aportes de Thorndike (Inteligencia Social), Wechsler (evaluación de factores no intelectuales), Leeper (pensamiento emocional), McClelland (con una nueva forma de pronosticar el éxito y desempeño laboral) y Gardner (inteligencias múltiples), se inició la construcción de una propuesta donde las habilidades no cognitivas son parte importante en el proceso de interacción social. Salovey y Mayer, a inicios de la década de 1990, dieron significado al constructo inteligencia emocional (Palomera, Fernández-Berrocal, \& Brackett, 2008), pero es Goleman quien permite su difusión en los campos científico, empresarial y educativo (Maidana \& Samudio, 2018).

Existen diversos modelos que estudian la inteligencia emocional. Sin embargo, la propuesta de Reuven Bar-On es calificada como un modelo mixto que considera habilidades emocionales y rasgos propios de la persona. La inteligencia emocional se constituye como un conjunto variado de habilidades que permite al individuo responder adecuadamente a las demandas del medio (Bar-On, 1997). Estas habilidades no cognoscitivas se agrupan en cinco componentes: intrapersonal, interpersonal, adaptabilidad, manejo de la tensión y el estado de ánimo en general. Este modelo multifactorial se relaciona con el potencial para el éxito; las habilidades emocionales influyen directamente en el bienestar de las personas y se desarrollan con la edad (Abanto, Higueras, \& Cueto, 2000; Bar-On, 2006).

Brasseur, Grégoire, Bourdu y Mikolajczak (2013) encontraron que las habilidades emocionales (especialmente la intrapersonal) se correlacionan positivamente con la edad. Luque-Reca, Augusto-Landa y Pulido-Martos (2016) reportaron altos niveles de inteligencia emocional en adultos mayores y Sharma (2017) explicó que la inteligencia emocional total se incrementa con la edad, pero recomienda implementar actividades de capacitación en la edad adulta para potenciar el desarrollado de las habilidades emocionales. En cuanto al sexo, las mujeres presentan mayor desarrollo de este tipo de habilidades en comparación con sus pares varones (Borsic \& Riveros, 2017; Danvila \& Sastre, 2010; Mandell \& Pherwani, 2003; Sánchez, Fernández-Berrocal, Montañés, \& Latorre, 2008).

Por otra parte, el "sentirse bien" es un aspecto de la vida sobre el cual se ha reflexionado a lo largo de la historia, pero el interés científico por estudiar el bienestar es reciente y toma importancia por sus efectos en la salud, relaciones sociales, desempeño académico, entre otros (Novoa \& Barra, 2015). El estudio del bienestar parte de dos perspectivas: la hedónica y eudaimónica; ambas con origen filosófico diferente. La perspectiva hedónica relaciona el bienestar con el goce y la felicidad, con experiencias placenteras; o también, un equilibrio entre los estímulos positivos y negativos. Desde el punto de vista eudaimónico, el bienestar va más allá de la felicidad personal; hace referencia al daimon, entendiéndose como potencial humano. Desde esta perspectiva, lo interesante es ver lo que la persona hace o piensa, y no tanto cómo se siente (Lent, 2004).

En la práctica, estas tradiciones filosóficas parece que se superponen y han permitido desarrollar diversos estudios y publicaciones sobre el bienestar subjetivo y psicológico (Keyes, Shmotkin, \& Ryff, 2002). El bienestar subjetivo se refiere a lo que sienten las personas sobre sus vidas y el placer por su existencia; mientras que el bienestar psicológico se relaciona con la valoración de aspectos que permite el desarrollo del potencial (Barrantes-Brais 
\& Ureña-Bonilla, 2015). El modelo de bienestar psicológico se basa en el supuesto de que los sujetos se esfuerzan por mejorar sus habilidades y desarrollar sus talentos; la evaluación del bienestar psicológico incluye la valoración de relaciones sociales de calidad, sentido de crecimiento y desarrollo (Gallagher, Lopez, \& Preacher, 2009). Las dimensiones hedónica y eudaimónica, frecuentemente, presentan una correlación moderada y alta (Ryff \& Keyes, 1995) por lo que, el bienestar debe ser estudiado como un fenómeno multidimensional; lo anterior se evidencia cuando una persona siente felicidad frente a actividades retadoras (Lent, 2004).

La satisfacción, desde el punto de vista holístico, es el producto final del conjunto de experiencias que una persona vive (Lounsbury, Park, Sundstrom, Williamson, \& Pemberton, 2004) y puede conceptualizarse como el bienestar psicológico (visión eudaimónica) que se obtiene al cotejar los objetivos alcanzados con las expectativas iniciales, es decir, se relaciona con la búsqueda y logro de aspiraciones personales (Medrano \& Pérez, 2010). De acuerdo con lo anterior, las experiencias que ofrece la universidad (académicas, físicas, espirituales y sociales) se convierten en referente directo para evaluar la satisfacción del estudiante (Inzunza et al., 2015).

El estudiante se siente satisfecho cuando ve que sus expectativas fueron atendidas o superadas (González-Peiteado, Pino-Juste, \& Penado-Abilleira, 2017). Por ello, el proceso educativo, la interacción con el docente y el conjunto de servicios disponibles en el campus, son elementos que el estudiante evalúa al momento de elaborar su opinión sobre la universidad. Como se observa, la satisfacción del estudiante posee diversas facetas de análisis, siendo la satisfacción académica una de las más importantes.

La satisfacción académica puede explicarse como el placer, gusto o deleite que siente el estudiante por sus quehaceres académicos dentro de una carrera, con la que se identifica y busca desarrollar sus habilidades (Bernal, Lauretti, \& Agreda, 2016). La satisfacción académica se ha convertido en un aspecto fundamental al momento de evaluar el proceso de adaptación, el índice de permanencia, la sensación de bienestar y el porcentaje de egresados universitarios (Merino-Soto, Dominguez-Lara, \& Fernández-Arata, 2017). Se ha observado, en estudiantes de ingeniería, que el deseo de permanecer en la misma carrera y culminar los estudios se relaciona con el logro de las metas y la satisfacción académica (Lent, Singley, Sheu, Schmidt, \& Schmidt, 2007).

Para conocer la satisfacción de los estudiantes, es necesario estudiar el rol del docente (Baños, Ortiz-Camacho, Baena-Extremera, \& Tristán-Rodríguez, 2017) y prestar atención a los vínculos interpersonales que se establecen entre ellos (Bernal et al., 2016). Como esta interacción configura un sistema integrado de vínculos humanos tan complejo que no se limita al plano cognitivo, las habilidades emocionales (empatía, solución de problemas, flexibilidad, tolerancia, optimismo, seguridad, entre otras) constituyen un aspecto del proceso educativo interesante de ser estudiado y que, según análisis previos, el docente universitario debe mostrar para ofrecer experiencias de aprendizaje gratificantes. Por otra parte, la satisfacción académica se ha convertido en una variable que, a través de los años, ha cobrado relevancia en las investigaciones realizadas en América Latina, entre ellos: Brasil, Chile, Argentina y Perú; ya que su análisis puede llevar a una mejor comprensión del bienestar estudiantil.

Como el estudiante es el centro del proceso educativo, el docente es parte fundamental en el aprendizaje y cumplimiento de expectativas, y las habilidades emocionales juegan un rol notable en la interacción docente-estudiante; se plantea como hipótesis que la inteligencia emocional del docente influye en los niveles de satisfacción académica, por lo que, el objetivo central de la presente investigación fue conocer la relación que existe entre la inteligencia emocional de los docentes, según el enfoque de Bar-On, y el nivel de satisfacción académica de los estudiantes universitarios. Además, se propuso estudiar la inteligencia emocional y la satisfacción académica según las características sociodemográficas de los docentes: sexo, edad y facultad en la que enseñan.

\section{Método}

\section{Diseño}

La presente investigación se enmarca en el enfoque cuantitativo. Asimismo, el diseño del estudio fue no experimental, de tipo correlacional. 


\section{Participantes}

Se evaluaron a dos grupos: uno conformado por docentes y el otro por estudiantes de diferentes carreras profesionales de una universidad privada de Lima, Perú. Los docentes fueron seleccionados mediante un muestreo por conveniencia, el cual permite seleccionar a los participantes de acuerdo con su accesibilidad y proximidad con los investigadores (Hernández, Fernández, \& Baptista, 2014). Para seleccionar la muestra de docentes, se tuvo como criterios de inclusión (1) estar trabajando un semestre en la universidad, (2) enseñar en los dos primeros años de facultad y (3) participar voluntariamente. Se obtuvo una muestra de 87 docentes con una edad promedio de 43.42 años ( $D E=6.07$ ), compuesta por $58.6 \%$ mujeres y $41.4 \%$ hombres. El $60.9 \%$ de los docentes pertenecían a la facultad de Humanidades (Comunicación y Psicología); mientras que el 39.1\% a la facultad de Ingeniería (Ingeniería Civil, Ingeniería de Sistemas e Ingeniería Industrial).

Con respecto al segundo grupo, la muestra estuvo conformada por estudiantes de primer y segundo año de facultad, inscritos en los cursos impartidos por los docentes que aceptaron participar en el estudio. Igual que en el grupo de docentes, la participación de los estudiantes en la investigación fue de forma voluntaria. El grupo estuvo conformado por un total de 597 estudiantes con matrícula vigente y asistencia regular, con edad promedio de 20.40 años ( $D E=2.05)$. En relación al género, esta muestra estuvo compuesta por un $49 \%$ mujeres y un 51\% hombres. Además, el 54\% pertenecían a la facultad de Humanidades, mientras que el $46 \%$ a la facultad de Ingeniería.

\section{Instrumentos}

Para medir la variable inteligencia emocional, se empleó la versión adaptada al contexto peruano del instrumento Bar-On Emotional Quotient Inventory (EQ-i), traducido al español como Inventario de Cociente Emocional de Bar-On (ICE). Esta adaptación fue realizada por Abanto et al. (2000), en una muestra de 1,246 personas (59.1\% hombres y $40.9 \%$ mujeres) pertenecientes a distintos niveles socioeconómicos y cuyas edades se registraron a partir de 16 años. Esta versión del instrumento está compuesta por 133 ítems agrupados en cinco componentes. Todos los ítems tuvieron cinco alternativas de respuesta desde 1 = "Rara vez o nunca es mi caso" a 5 = "Con mucha frecuencia o siempre es mi caso". Además, el instrumento presenta una escala de medición que permite interpretar los puntajes obtenidos como "nivel de desarrollo" desde baremos peruanos (ver Tabla 1).

Abanto et al. (2000) explica que, como parte del desarrollo del ICE de Bar-On, este instrumento fue sometido a diferentes pruebas de validez. Para determinar la validez estructural del instrumento se realizó un análisis factorial, del cual se desprende la existencia de equilibrio en la estructura factorial 1 - 5 - 15 (CE total - escalas compuestas

\section{Tabla 1}

Inteligencia Emocional: componentes y pauta interpretativa

\begin{tabular}{|l|l|l|}
\hline Variable & Componentes & Niveles \\
\hline & Componente Intrapersonal & 130 a + (Marcadamente alta) \\
& Componente Interpersonal & $120-129$ (Muy alta) \\
Inteligencia & Componente de Adaptabilidad & $110-119$ (Alta) \\
& & $90-109$ (Promedio) \\
& Componente de Manejo de la Tensión & $80-89$ (Baja) \\
& Componente de Estado de Ánimo General & $70-79$ (Muy baja) \\
& & Por debajo de 70 (Marcadamente baja) \\
\hline
\end{tabular}


- subescalas). Asimismo, se ha demostrado una adecuada validez convergente al correlacionar la escala con constructos como enfrentamiento de situaciones tensionantes, desempeño laboral y satisfacción con el trabajo. De igual forma, la validez divergente fue corroborada al correlacionar el ICE de Bar-On con constructos tales como autoestima, sumisión, conformismo, inestabilidad, despreocupación, etc. Además, a través de la validez discriminante, se evidenció que el ICE puede evaluar y demostrar diferencias entre personas que gestionan su inteligencia emocional de aquellas que no la han desarrollado. Para el empleo de la versión adaptada del ICE de Bar-On, en la presente investigación se revisó la fiabilidad de las puntuaciones a través de la consistencia interna de la escala y sus dimensiones. En la Tabla 2, se puede observar los coeficientes de Alfa obtenidos, los cuales indican que las puntuaciones producidas por el instrumento son altamente confiables.

El segundo instrumento mide la Satisfacción Académica. La versión original fue construida para medir la satisfacción en estudiantes universitarios brasileños (Sisto et al., 2008). Esta escala estuvo conformada por 35 ítems agrupados en cuatro dimensiones: percepción del ambiente pedagógico, percepción de la afectividad, la percepción del ambiente físico y la percepción del ajuste social; y las respuestas se registraban a través de una escala Likert de cuatro opciones: "nunca" (0) hasta "siempre" (3), donde un mayor puntaje indi- ca una mayor satisfacción. La validez de constructo fue demostrada a través del análisis factorial y se calcularon los índices de consistencia interna, donde obtuvo los siguientes coeficientes de Alfa .86 (escala total), .87 (percepción del entorno de enseñanza), .76 (percepción de la afectividad), .73 (percepción del ambiente físico) y, .72 (percepción del ajuste social).

Posteriormente, Medrano y Pérez (2010) realizaron una adaptación al español de la dimensión percepción del entorno de enseñanza en estudiantes universitarios argentinos. Dichos autores decidieron trabajar con esta dimensión debido a dos razones: el primer motivo fue empírico, ya que se trata del factor que explica la mayor variabilidad del instrumento (15.1\%). El segundo motivo fue teórico, debido a que los ítems que componen la escala de satisfacción con el entorno de enseñanza son más congruentes con la propuesta de Lent en 2004. En esta adaptación participaron 251 estudiantes de diferentes carreras y se realizaron estudios de validez de constructo y consistencia interna utilizando análisis factorial exploratorio y coeficiente Alfa, respectivamente. Los resultados obtenidos sugirieron una estructura interna unidimensional compuesta por ocho ítems que explicaba un $49 \%$ de la variabilidad de respuesta, así como una elevada homogeneidad ( $\alpha=$.84) de la escala.

Para la presente investigación se realizaron cambios en la redacción de los ítems 3 y 7, de "Me gustan mis profesores" a "Me gusta mi profesor"

Tabla 2

Índice de Confiabilidad de ICE y sus Componentes

\begin{tabular}{|c|c|c|c|}
\hline \multirow{2}{*}{ Escala } & \multirow[b]{2}{*}{$\alpha$} & \multicolumn{2}{|c|}{ IC $95 \%$} \\
\hline & & $\mathbf{L i}$ & Ls \\
\hline Escala general & .95 & .93 & .97 \\
\hline Componente intrapersonal & .88 & .84 & .92 \\
\hline Componente interpersonal & .81 & .74 & .87 \\
\hline Componente de adaptabilidad & .80 & .73 & .86 \\
\hline Componente del manejo de la tensión & .76 & .68 & .83 \\
\hline Componente estado de ánimo general & .77 & .69 & .84 \\
\hline
\end{tabular}

Nota. Li: Límite inferior del coeficiente alfa, Ls: Límite superior del coeficiente alfa 
y "Los profesores son abiertos al diálogo" a "El profesor está abierto al diálogo". Se realizaron estas modificaciones con el propósito de centrar la evaluación de la satisfacción académica en el entorno pedagógico creado por el docente evaluado. Con el fin de demostrar validez de constructo, se realizó un análisis factorial exploratorio donde la medida de adecuación muestral de Kaiser Meyer-Olkin (KMO) obtuvo un valor adecuado de .849; además, la prueba de esfericidad de Bartlett fue significativa $\left(X^{2}=756.739 ; p \leq .001\right)$; estos resultados mostraron que se cumplieron con las condiciones básicas para continuar con el análisis. Posteriormente, se realizó la extracción de factores empleando el método de máxima verosimilitud con rotación Oblimín. Tras esto se confirmó que los ocho ítems del instrumento se agrupan en una sola dimensión que explica el 31\% de la varianza de las respuestas, las cargas factoriales de los ítems estuvieron entre .51 y .60. Con respecto a la confiabilidad, en el presente estudio se obtuvo un coeficiente Alfa .78 (con intervalos de confianza al 95\% de .74 a .81). Este resultado indica que las puntuaciones son fiables.

\section{Procedimientos}

Se aplicó el ICE a los docentes dos semanas antes de terminar el semestre. La aplicación de la escala fue realizada por los investigadores, quienes entregaron el cuestionario impreso junto con el consentimiento informado. El llenado de ambos documentos duró en promedio 42 minutos por persona. La población total estuvo constituida por 98 docentes, de los cuales cuatro rechazaron expresamente participar en la investigación. El instrumento fue aplicado a un total de 94 docentes. Sin embargo, solo 87 cumplieron con los criterios de validez de la prueba de ICE de Bar-On (índice de inconsistencia, impresión negativa, impresión positiva e ítem 133 de validez general), lo que representa el 88.8\% de la población.

Por otro lado, la aplicación del instrumento que mide la satisfacción académica en los estudiantes se llevó a cabo durante las dos últimas semanas del semestre, en las cuales se accedió a los últimos 15 minutos de clases para explicar el objetivo de la investigación y consultar si deseaban participar voluntariamente. Si accedían, los investigadores entregaban el cuestionario impreso junto al con- sentimiento informado. De esta forma, se logró acceder a una muestra de 597 estudiantes. La aplicación de la escala de satisfacción académica duró aproximadamente seis minutos por estudiante.

\section{Análisis de datos}

Por la naturaleza de la investigación, se calculó el promedio de la variable satisfacción académica del estudiante respecto a cada docente. De esta forma, cada docente participante obtuvo dos calificaciones: una perteneciente a la variable inteligencia emocional y otra a la variable satisfacción académica.

Para la realización de los análisis estadísticos pertinentes se empleó el paquete estadístico SPSS v.22. Primero, se realizó el análisis de normalidad a las distribuciones de las puntuaciones por escalas, sub-escalas y a las características sociodemográficas. Se empleó la prueba de Kolmogorov-Smirnov para las puntuaciones totales, mientras que para las puntuaciones divididas por las características sociodemográficas de los docentes se utilizó la prueba de Shapiro-Wilk. Con estas pruebas se reportó falta de normalidad en algunas sub-escalas, por lo cual, se procedió a analizar los índices de asimetría y curtosis, los cuales fueron menores a 3 y 10 respectivamente. Esto permitió señalar que las distribuciones de los datos se encontraban dentro de un rango adecuado (Kline, 2011) y se podía ejecutar los análisis paramétricos correspondientes.

Con el propósito de responder al objetivo central de la investigación, se correlacionó la inteligencia emocional del docente con el promedio de la satisfacción académica del alumno respecto al docente evaluado. De esta forma, se obtuvo dos puntuaciones por docente, lo cual permitió realizar los análisis correlacionales mediante el coeficiente $r$ de Pearson. También se correlacionó el promedio por docente de la satisfacción académica de los estudiantes con las características sociodemográficas del docente mediante el coeficiente biserial puntual. Se realizaron comparaciones de medias empleando el estadístico $t$ Student para la inteligencia emocional y la satisfacción académica del estudiante por docente según las características sociodemográficas del educador. Asimismo, se empleó ANOVA de un factor para comparar la satisfacción académica según el nivel de inteligencia emocional de docente. 


\section{Resultados}

Se halló que el promedio del cociente emocional (CE) en los docentes se encuentra en un nivel promedio (entre 90 y 109) de acuerdo con las pautas interpretativas de la adaptación al contexto peruano del ICE de Barón (Abanto et al., 2000). En la tabla 3 se aprecia que 91\% de los docentes evaluados está en los niveles promedio, alto y muy alto. Esto indica que la mayoría de los sujetos presentan una capaci- dad emocional entre adecuada y bien desarrollada.

En la Tabla 4, se presentan las principales medidas descriptivas de las puntuaciones de inteligencia emocional general y sus dimensiones. Se observa que en todos los casos los puntajes promedios son superiores a cien.

Para responder el objetivo central de la investigación, se relacionó la inteligencia emocional y sus componentes con la satisfacción académica. Se encontraron correlaciones estadísticamente significativas en todos los casos (ver Tabla 5).

Tabla 3

Nivel de Inteligencia Emocional

\begin{tabular}{|c|c|c|}
\hline Niveles & $f$ & $\%$ \\
\hline Marcadamente Alta & 0 & 0 \\
\hline Muy Alta & 11 & 13 \\
\hline Alta & 20 & 23 \\
\hline Promedio & 48 & 55 \\
\hline Baja & 5 & 6 \\
\hline Muy Baja & 3 & 3 \\
\hline Marcadamente Baja & 0 & 0 \\
\hline
\end{tabular}

Nota. $f$ : frecuencia

Tabla 4

Medidas Descriptivas Inteligencia Emocional y sus Dimensiones

\begin{tabular}{|l|c|c|c|c|}
\hline Variables & $\boldsymbol{M}$ & $\boldsymbol{D E}$ & $\boldsymbol{M i ́ n}$ & Máx \\
\hline Inteligencia emocional total & 105.81 & 12.16 & 78 & 124 \\
\hline Intrapersonal & 100.13 & 12.47 & 65 & 118 \\
\hline Interpersonal & 107.55 & 12.40 & 85 & 126 \\
\hline Adaptabilidad & 105.52 & 12.02 & 79 & 126 \\
\hline Manejo de Tensión & 102.61 & 10.27 & 83 & 124 \\
\hline Ánimo en General & 108.00 & 12.18 & 75 & 128 \\
\hline
\end{tabular}

Nota. M: Media, DE: Desviación estándar, Min: Puntaje mínimo, Max: Puntaje máximo

Tabla 5

Correlación entre Inteligencia Emocional del Docente y Satisfacción Académica ( $n=87)$

\begin{tabular}{|l|c|c|c|c|c|c|}
\hline & CE Total & Intrapersonal & Interpersonal & Adaptabilidad & $\begin{array}{c}\text { Manejo de } \\
\text { Tensión }\end{array}$ & $\begin{array}{c}\text { Ánimo en } \\
\text { General }\end{array}$ \\
\hline Satisfacción Académica & $.80^{*}$ & $.70^{*}$ & $.73^{*}$ & $.64^{*}$ & $.72^{*}$ & $.66^{*}$ \\
\hline
\end{tabular}

Nota: Se empleó el coeficiente de Pearson para las correlaciones debido a la normalidad de las subescalas. * $<.001$ 
Para propósitos de la investigación, los docentes fueron agrupados por su edad -30 a 44 años y de 45 años a más-; y según la facultad donde enseñan -Humanidades (Comunicación y Psicología) e Ingeniería (Ingeniería Civil, Ingeniería Industrial, Ingeniería de Sistemas)-. Posteriormente, se correlacionó la variable satisfacción académica con las características sociodemográficas de los docentes (sexo, edad y facultad en la que enseñan). Se obtuvieron correlaciones directas en todos los análisis. Sin embargo, la única relación estadísticamente significativa fue entre las variables edad del docente y satisfacción académica. Esta relación obtuvo un coeficiente de correlación igual a .53 ( $\mathrm{p}$ < .01), lo que indicaría que una mayor satisfacción académica de los estudiantes estaría asociada a una mayor edad en los docentes.
Por otro lado, se realizaron contrastes de medias de inteligencia emocional y sus componentes según las características sociodemográficas de los docentes. Con respecto al sexo del docente, no se encontraron diferencias estadísticamente significativas (ver Tabla 6).

En la tabla 7, se presenta la comparación de los puntajes obtenidos en Inteligencia Emocional, según la edad de los docentes. Los resultados muestran que los docentes de mayor edad presentan, en promedio, mayor inteligencia emocional que los docentes más jóvenes. Esta tendencia se repite en cada uno de los componentes.

Además, según la facultad, se aprecia un mayor puntaje en el componente interpersonal de los docentes de Humanidades (ver Tabla 8). Alcanzan valores estadísticamente significativos solo en esta dimensión de la Inteligencia Emocional.

\section{Tabla 6}

Comparación de la Inteligencia Emocional, según el sexo de los docentes

\begin{tabular}{|c|c|c|c|c|c|}
\hline & Sexo & $M$ & $D E$ & $t$ & $d$ \\
\hline \multirow{2}{*}{ CE Total } & Mujeres ( $\mathrm{n}=51)$ & 107.61 & 12.02 & \multirow{2}{*}{0.97} & \multirow{2}{*}{0.35} \\
\hline & Hombres $(\mathrm{n}=36)$ & 103.31 & 12.39 & & \\
\hline \multirow{2}{*}{ Intrapersonal } & Mujeres ( $n=51)$ & 101.28 & 11.76 & \multirow{2}{*}{0.60} & \multirow{2}{*}{0.21} \\
\hline & Hombres $(n=36)$ & 98.54 & 13.72 & & \\
\hline \multirow{2}{*}{ Interpersonal } & Mujeres $(n=51)$ & 108.39 & 12.48 & \multirow{2}{*}{0.44} & \multirow{2}{*}{0.16} \\
\hline & Hombres $(n=36)$ & 106.38 & 12.69 & & \\
\hline \multirow[b]{2}{*}{ Adaptabilidad } & Mujeres $(n=51)$ & 106.39 & 10.81 & \multirow[b]{2}{*}{0.47} & \multirow[b]{2}{*}{0.17} \\
\hline & Hombres $(n=36)$ & 104.31 & 13.89 & & \\
\hline \multirow[b]{2}{*}{ Manejo de Tensión } & Mujeres ( $\mathrm{n}=51)$ & 104.11 & 10.21 & \multirow[b]{2}{*}{0.95} & \multirow[b]{2}{*}{0.35} \\
\hline & Hombres $(n=36)$ & 100.54 & 10.39 & & \\
\hline \multirow[b]{2}{*}{ Ánimo en General } & Mujeres ( $\mathrm{n}=51)$ & 110.72 & 8.85 & \multirow[b]{2}{*}{1.38} & \multirow[b]{2}{*}{0.52} \\
\hline & Hombres $(n=36)$ & 104.23 & 15.28 & & \\
\hline
\end{tabular}


Tabla 7

Comparación de la Inteligencia Emocional según la edad de los docentes

\begin{tabular}{|c|c|c|c|c|c|}
\hline & Grupo de edad & $M$ & $D E$ & $t$ & $d$ \\
\hline \multirow{2}{*}{ CE Total } & 30 a $44(n=51)$ & 100 & 11.49 & \multirow{2}{*}{$-3.75^{*}$} & \multirow{2}{*}{1.41} \\
\hline & 45 años a más $(\mathrm{n}=36)$ & 113.85 & 7.88 & & \\
\hline \multirow{2}{*}{ Intrapersonal } & 30 a $44(n=51)$ & 95.11 & 12.46 & \multirow{2}{*}{$-2.96^{*}$} & \multirow{2}{*}{1.11} \\
\hline & 45 años a más $(\mathrm{n}=36)$ & 107.08 & 8.87 & & \\
\hline \multirow{2}{*}{ Interpersonal } & 30 a $44(n=51)$ & 101.89 & 11.2 & \multirow{2}{*}{$-3.51^{*}$} & \multirow{2}{*}{1.29} \\
\hline & 45 años a más ( $\mathrm{n}=36)$ & 115.38 & 9.59 & & \\
\hline \multirow{2}{*}{ Adaptabilidad } & 30 a $44(n=51)$ & 100.17 & 12.08 & \multirow{2}{*}{$-3.39^{*}$} & \multirow{2}{*}{1.28} \\
\hline & 45 años a más (n=36) & 112.92 & 7.23 & & \\
\hline \multirow{2}{*}{ Manejo de Tensión } & 30 a $44(n=51)$ & 98.11 & 9.36 & \multirow{2}{*}{$-3.32 *$} & \multirow{2}{*}{1.22} \\
\hline & 45 años a más (n=36) & 108.85 & 8.19 & & \\
\hline \multirow{2}{*}{ Ánimo en General } & 30 a $44(n=51)$ & 103.11 & 12.61 & \multirow{2}{*}{$-2.95^{*}$} & \multirow{2}{*}{1.11} \\
\hline & 45 años a más (n=36) & 114.77 & 7.88 & & \\
\hline
\end{tabular}

Nota. ${ }^{*} p<0.05$

Tabla 8

Comparación de la Inteligencia Emocional según con la facultad en la que enseñan los docentes

\begin{tabular}{|c|c|c|c|c|c|}
\hline & Facultad & $M$ & $D E$ & $t$ & $d$ \\
\hline \multirow{2}{*}{ CE Total } & Ingeniería $(n=34)$ & 104 & 12.49 & \multirow{2}{*}{-1.04} & \multirow{2}{*}{0.39} \\
\hline & Humanidades $(\mathrm{n}=53)$ & 108.67 & 11.56 & & \\
\hline \multirow{2}{*}{ Intrapersonal } & Ingeniería ( $\mathrm{n}=34$ ) & 98 & 12.98 & \multirow{2}{*}{-1.21} & \multirow{2}{*}{0.45} \\
\hline & Humanidades $(n=53)$ & 103.5 & 11.32 & & \\
\hline \multirow{2}{*}{ Interpersonal } & Ingeniería ( $\mathrm{n}=34$ ) & 103.68 & 11.18 & \multirow{2}{*}{$-2.34^{*}$} & \multirow{2}{*}{0.85} \\
\hline & Humanidades $(\mathrm{n}=53)$ & 113.67 & 12.17 & & \\
\hline \multirow{2}{*}{ Adaptabilidad } & Ingeniería $(n=34)$ & 105.16 & 12.97 & \multirow{2}{*}{-0.21} & \multirow{2}{*}{0.08} \\
\hline & Humanidades $(n=53)$ & 106.08 & 10.87 & & \\
\hline \multirow{2}{*}{ Manejo de Tensión } & Ingeniería $(\mathrm{n}=34)$ & 101.68 & 10.79 & \multirow{2}{*}{-0.63} & \multirow{2}{*}{0.23} \\
\hline & Humanidades $(\mathrm{n}=53)$ & 104.08 & 9.66 & & \\
\hline \multirow{2}{*}{ Ánimo en General } & Ingeniería $(\mathrm{n}=34)$ & 107.74 & 13.62 & \multirow{2}{*}{-0.15} & \multirow{2}{*}{0.06} \\
\hline & Humanidades $(\mathrm{n}=53)$ & 108.42 & 10.03 & & \\
\hline
\end{tabular}

Nota. ${ }^{*} p<0.05$ 
Asimismo, se realizaron contrastes de medias de la variable satisfacción académica con relación a los niveles de CE Total. Para efectos de este análisis, se decidió agrupar los resultados en tres niveles: Nivel alto (niveles marcadamente alto, muy alto y alto), Nivel promedio (nivel promedio) y Nivel Bajo (niveles bajo, muy bajo y marcadamente bajo). En la Tabla 9 se observa que existen diferencias de satisfacción académica según los niveles de CE de los docentes. Se puede apreciar que los docentes con mayor nivel de inteligencia emocional presentan una mayor satisfacción académica por parte de los estudiantes (ver Tabla 9).

Además, se realizaron contrastes de medias de la variable satisfacción académica en relación con las características sociodemográficas del docente (ver Tabla 10). Tras realizar el análisis, se encontró solo una diferencia significativa con relación a la edad del docente. Estos resultados parecen indicar que un mayor nivel de satisfacción académica en los estudiantes se relaciona directamente con la edad del docente.

\section{Discusión}

En general, se encontró que los docentes universitarios presentan una inteligencia emocional promedio. Estos resultados se condicen con los hallazgos de Carcausto (2016) en Lima y Contreras y Dextre (2016) en Huancayo. Solo un 9\% de docentes presenta un nivel bajo o muy bajo de inteligencia emocional. Según la normativa legal vigente y el reglamento interno de la universidad, el ingreso a la carrera docente depende del grado académico obtenido (como requisito base se exige el grado de maestro/magíster), demostrar experiencia docente no menor de tres años, superar los filtros psicológicos y de habilidades blandas que evalúa el departamento de desarrollo docente, así como una rigurosa entrevista y clase modelo. El cumplimiento de estos requisitos hace posible que la plana docente haya sido evaluada, indirectamente, en habilidades que pertenecen al constructo inteligencia emocional. De esta forma, se podría explicar los niveles

\section{Tabla 9}

Comparación de la Satisfacción Académica de los estudiantes, según el nivel de Inteligencia Emocional

\begin{tabular}{|c|c|c|c|c|c|c|c|c|}
\hline \multirow{3}{*}{$\begin{array}{l}\text { Satisfacción } \\
\text { Académica }\end{array}$} & \multicolumn{2}{|c|}{$\begin{array}{l}\text { Nivel alto } \\
(n=37)\end{array}$} & \multicolumn{2}{|c|}{$\begin{array}{l}\text { Nivel promedio } \\
\qquad(\mathrm{n}=42)\end{array}$} & \multicolumn{2}{|c|}{$\begin{array}{l}\text { Nivel bajo } \\
\qquad(\mathrm{n}=8)\end{array}$} & \multirow{2}{*}{$F$} & \multirow{2}{*}{$p$} \\
\hline & $M$ & $D E$ & $M$ & $D E$ & $M$ & $D E$ & & \\
\hline & 20.16 & 1.33 & 18.78 & 0.57 & 17.18 & 1.30 & 38.6 & .000 \\
\hline
\end{tabular}

Tabla 10

Comparación de la Satisfacción Académica según características sociodemográfica del docente

\begin{tabular}{|c|c|c|c|c|}
\hline & & $M$ & $D E$ & $t$ \\
\hline \multirow{2}{*}{ Sexo } & Mujeres $(n=51)$ & 19.05 & 1.27 & \multirow{2}{*}{-0.29} \\
\hline & Hombres $(n=36)$ & 19.19 & 1.45 & \\
\hline \multirow{2}{*}{ Edad } & 30 a 44 años $(n=51)$ & 18.53 & 0.97 & \multirow{2}{*}{$-3.36^{*}$} \\
\hline & 45 a más ( $n=36)$ & 19.93 & 1.36 & \\
\hline \multirow{2}{*}{ Facultad } & Humanidades $(\mathrm{n}=53)$ & 19.35 & 1.1 & \multirow{2}{*}{-0.80} \\
\hline & Ingeniería $(n=34)$ & 18.96 & 1.46 & \\
\hline
\end{tabular}

Nota. ${ }^{*} p<0.05$ 
de inteligencia emocional obtenidos por los docentes que participaron en este estudio.

Los resultados muestran que existe correlación positiva entre la inteligencia emocional del docente y la satisfacción académica del estudiante. Asimismo, se observa que la correlación entre satisfacción académica y el componente interpersonal (relaciones interpersonales, responsabilidad social y empatía) es mayor respecto de las correlaciones con los otros componentes. Sin embargo, es importante mencionar que los componentes manejo de la tensión e intrapersonal también obtienen índices de correlación positivos y altos con la satisfacción académica. Lo anterior parece coincidir con lo planteado por Fernández et al. (2009) sobre la empatía y las competencias socioafectivas; con la propuesta de Jennings y Greenberg (2009) y con lo afirmado con Thomson y Palermo (2014) sobre las emociones y su impacto en el desempeño del docente; con el aporte de Pertegal-Felices et al. (2011) sobre la relación entre la calidad del proceso educativo y las competencias emocionales del docente; con el planteamiento de Almiron y Porro (2014) sobre el rol de la empatía en el éxito del docente en clase; con lo planteado por Golombek y Doran (2014) sobre el vínculo de las emociones y el proceso de enseñanza; y los hallazgos de Palomera et al. (2017) sobre las habilidades emocionales como promotoras de la creación de ambientes efectivos de aprendizaje.

Si bien los antecedentes no relacionan directamente las variables del presente estudio, los hallazgos expuestos refuerzan la importancia de la inteligencia emocional del docente en el proceso educativo y en la interacción docente-estudiante. Las habilidades emocionales, específicamente las interpersonales, permiten al docente construir y mantener en el tiempo relaciones de entendimiento mutuo con los estudiantes, pertenecer al grupo y demostrar cooperación por el bien de todos, comprender y apreciar las emociones y sentimientos, comprometerse con el desempeño académico de los estudiantes y crear ambientes adecuados para el aprendizaje y la satisfacción académica. Se podría decir que el desarrollo de habilidades emocionales en el docente se traduce en indicadores positivos de la satisfacción académica.

Asimismo, es importante prestar atención al ni- vel de correlación entre la satisfacción académica y el componente manejo de la tensión (segundo índice de correlación más alto). Esto indicaría que la capacidad de manejar situaciones difíciles y resistir a eventos adversos sin agobiarse (gestión de la agresión y conductas irresponsables) son factores que los estudiantes perciben como positivos y que contribuyen al desarrollo de las clases. En el mismo sentido, el índice de correlación entre la satisfacción académica y el componente intrapersonal (tercer índice más alto) indicaría que los estudiantes valoran positivamente que el docente reconozca sus propias emociones, defienda sus pensamientos, se respete a sí mismo, evidencie deseos de superación y muestre confianza en sí mismo.

Observando los resultados de la inteligencia emocional por sexo, se ha encontrado que las docentes mujeres obtienen un puntaje mayor que sus colegas varones en el CE total y en cada uno de los componentes emocionales. A primera vista, estos hallazgos parecen coincidir con Mandell y Pherwani (2003) en Estados Unidos, Borsic y Riveros (2017) en México y con Sánchez et al. (2008) en España sobre el mayor índice de inteligencia emocional que tendrían las mujeres en comparación con los hombres. Sin embargo, según la evidencia del presente estudio y el contraste de medias, no se reportan diferencias significativas entre la inteligencia emocional y el sexo de los docentes, ni en el CE total ni en sus componentes. Esto último coincide con la propuesta de Danvila y Sastre (2010) sobre los niveles de inteligencia emocional. Los autores explican que entre hombres y mujeres no existen diferencias en las puntuaciones globales, pero es posible que las diferencias sean notorias al momento de poner en práctica dichas habilidades, por ejemplo, en el caso de la empatía.

Según los resultados, los docentes de 45 años a más presentan mayor inteligencia emocional en comparación con los de menor edad (de 30 a 44 años). Estos resultados coinciden con la propuesta de Abanto, et al. (2000), Bar-On (2006), Brasseur et al. (2013), Luque-Reca et al. (2016) y Sharma (2017), quienes exponen que la inteligencia emocional se correlaciona con la edad. Sin embargo, Borsic y Riveros (2017) encontraron que los adultos jóvenes (25 a 43 años) poseen mayor inteligencia emocional que los docentes comprendidos entre 44 y 51 
años, mientras que Danvila y Sastre (2010) plantean que esta relación no siempre es positiva, ya que a cierta edad puede disminuir. La evidencia estadística del estudio muestra que existen diferencias significativas entre la edad de los docentes y el CE total, y también en cada uno de sus componentes. Esto confirma que los docentes de 45 años a más presentan mayor inteligencia emocional, lo que se traduce en un aporte a lo propuesto por Abanto et al. (2000) y Bar-On (2006). Como afirman Danvila y Sastre (2010), la experiencia del docente, que generalmente aumenta con la edad, se convierte en un factor que contribuye al desarrollo de la inteligencia emocional, por lo que no se puede asegurar que la edad sea el único agente responsable de dicho desarrollo.

En el CE total y en cada uno de sus componentes, los docentes de la facultad de Humanidades obtuvieron mayor puntaje respecto de los docentes de Ingeniería. Sin embargo, solo se ha reportado diferencia significativa en el componente interpersonal. Este resultado parece indicar que se debe trabajar con estos docentes para mejorar sus habilidades emocionales y también analizar su proceso formativo universitario. En esta línea, el trabajo de Neri y Hernández (2019), desarrollado en México, reportó que el 39\% de estudiantes de Ingeniería presenta problemas para relacionarse con los demás y que el 12\% manifestó que no poseía dicha competencia. Según el trabajo de Marzo, Iglesias y Torres (2006), la formación de los ingenieros no responde a las demandas del mercado laboral, se presenta una situación de déficit de habilidades comunicativas, trabajo en equipo y liderazgo. Villa-Peralta (2017) recomienda que la formación de los ingenieros debe enmarcarse dentro de las exigencias del mundo contemporáneo; Zepeda-Hurtado, Cardoso-Espinosa y Rey-Benguría (2019) proponen la creación de un perfil híbrido que desarrolle el aspecto técnico-científico y también habilidades comunicativas y blandas.

Los resultados indican que los docentes con mayor inteligencia emocional reportan mayor satisfacción en los estudiantes. Es poco probable que la inteligencia emocional sea la única variable que pueda explicar dichas puntuaciones en la satisfacción académica, ya que como menciona Abanto et al. (2000), la inteligencia emocional está relacionada con otras variables como la capa- cidad intelectual cognoscitiva, factores personales de la conducta, entre otros. Lo cierto es que, según la evidencia, la inteligencia emocional puede ser un indicador para evaluar el potencial de éxito en el desempeño de los docentes universitarios. Además, se ha encontrado diferencias significativas en la satisfacción académica cuando se comparan los resultados según la edad. Los docentes de mayor edad parecen lograr mayor nivel de satisfacción académica, de allí la importancia de la experiencia profesional y la experiencia de vida.

A manera de conclusión, y según los resultados expuestos, la relación entre la inteligencia emocional del docente y la satisfacción académica del estudiante es positiva. No se ha encontrado evidencia para asegurar diferencias en la inteligencia emocional según el sexo de los docentes, pero sí en cuanto a la edad. Los docentes de la facultad de Humanidades presentan mayores habilidades interpersonales y se evidencian puntajes altos de satisfacción académica en aquellos estudiantes con docentes de mayor edad.

De acuerdo con todo lo anterior, se recomienda explicitar la evaluación de la variable inteligencia emocional en el proceso de selección de los docentes universitarios, específicamente en el componente interpersonal, debido a que este conjunto de habilidades entra en ejecución al momento de interactuar con los estudiantes y, al parecer, se relaciona significativamente con la satisfacción académica. Asimismo, el área de Calidad Educativa, junto con el departamento de Desarrollo Docente de cada universidad, debería promover el desarrollo de las habilidades emocionales en los profesionales que están ejerciendo la docencia actualmente. Esto con el objetivo de mejorar la formación emocional del personal y promover el empleo de este tipo de habilidades durante el proceso de enseñanza-aprendizaje. También sería aconsejable revisar la malla curricular, no solo de las carreras de Humanidades e Ingeniería, sino de todas las carreras a nivel nacional, para asegurar que los futuros profesionales, y también futuros docentes universitarios, desarrollen las habilidades técnico-científico propias de la carrera, así como habilidades emocionales que permitan, según Abanto et al. (2000), un manejo exitoso de las exigencias del entorno.

Por la naturaleza del diseño no experimental y 
correlacional del estudio, no se ha podido demostrar una relación causa-efecto entre la inteligencia emocional del docente y la satisfacción académica del estudiante. Además, no se han controlado otras variables como el tipo de personalidad, la inteligencia cognoscitiva del docente y del estudiante, los años de experiencia profesional o en cátedra del docente, el éxito obtenido en la profesión, etc.

Como los resultados no son determinantes, se recomienda promover nuevas investigaciones a fin de lograr una mayor comprensión sobre la relación que existiría entre la inteligencia emocional y la satisfacción académica. Por ejemplo, sería interesante replicar este estudio en otras universidades para comparar los resultados, incorporar la experiencia profesional del docente como variable para estudiar su comportamiento junto a la inteligencia emocional y la satisfacción académica, adoptar un enfoque mixto para el estudio de la variable inteligencia emocional. De esta forma, podría explicarse, por ejemplo, la diferencia encontrada en los puntajes de los docentes según la facultad y explorar a profundidad las similitudes y diferencias que se manifiestan en el desarrollo y empleo de estas habilidades según el sexo de los participantes. También sería recomendable investigar la relación entre la inteligencia emocional del docente y el deseo de los estudiantes de mantenerse en la institución, en la carrera y culminarla; ya que estas aspiraciones se relacionan con buenos niveles de satisfacción académica en estudiantes de Ingeniería, según los datos que muestra Lent et al. (2007).

A pesar de las limitaciones, los hallazgos del presente estudio contribuyen a ampliar el conocimiento sobre la interacción docente-estudiante a nivel universitario, convirtiéndose en un antecedente para futuras investigaciones sobre las habilidades emocionales, su impacto en el entorno de aprendizaje universitario y en la satisfacción académica. Además, se configura en un esfuerzo académico que puede contribuir a la elaboración de un perfil emocional del docente universitario.

\section{Referencias}

Abanto, Z., Higueras, L., \& Cueto, J. (2000). ICE Inventario de cociente emocional de Baron (test para la medida de la inteligencia emocional Reuven Bar-On Ph.D.). Lima:
Grafimag S.R.L.

Almiron, M., \& Porro, S. (2014). Las TIC en la enseñanza: un análisis de casos. Revista Electrónica de Investigación Educativa, 16(2), 152-160. Recuperado de http://www. scielo.org.mx/pdf/redie/v16n2/v16n2a10.pdf

Baños, R., Ortiz-Camacho, M., Baena-Extremera, A., \& Tristán-Rodríguez, J. (2017). Satisfacción, motivación y rendimiento académico en estudiantes de Secundaria y Bachillerato: antecedentes, diseño, metodología y propuesta de análisis para un trabajo de investigación. Espiral. Cuadernos del Profesorado, 10(20), 4050. https://doi.org/10.25115/ecp.v10i20.1011

Barrantes-Brais, K., \& Ureña-Bonilla, P. (2015). Bienestar psicológico y bienestar subjetivo en estudiantes universitarios costarricenses. Revista Intercontinental de Psicología y Educación, 17(1), 101-123. Recuperado de http://www.redalyc.org/articulo.oa?id=80242935006

Bar-On, R. (1997). BarOn emotional quotient inventory: Technical manual. Toronto: Multi-Health Systems Inc.

Bar-On, R. (2006). The Bar-On model of emotional-social intelligence (ESI). Psicothema, 18, supl., 13-25. Recuperado de http://www.eiconsortium.org/pdf/baron_model of_emotional_social_intelligence.pdf

Bernal, J., Lauretti, P., \& Agreda, M. (2016). Satisfacción académica en estudiantes de la Facultad de Ingeniería de la Universidad del Zulia. Multiciencias, 16(3), 301-309. Recuperado de http://www.redalyc.org/ pdf/904/90453464009.pdf

Borsic, Z. D., \& Riveros, A. (septiembre, 2017). Inteligencia emocional en el desempeño laboral de docentes de instituciones de educación superior. En J. S. Méndez (Presidencia), XXII Congreso internacional de contaduría, administración e informática. Conferencia llevada a cabo en la UNAM, Ciudad de México, México. Recuperado de http://congreso.investiga.fca.unam.mx/docs/ xxii/docs/3.04.pdf

Brasseur, S., Grégoire, J., Bourdu, R., \& Mikolajczak, M. (2013). The Profile of Emotional Competence (PEC): Development and Validation of a Self-Reported Measure that Fits Dimensions of Emotional Competence Theory. PLOS ONE, 8(5). doi:10.1371/journal.pone.0062635

Buitrago, R., Ávila, A., \& Cárdenas, R. (2017). El sentido y el significado atribuido a las emociones por el profesorado en formación de la Universidad Pedagógica y Tecnológica de Colombia. Contextos Educativos. Revista de Educación, O(20), 77-93. http://doi. org/10.18172/con.2998

Carcausto, F. D. (2016). La inteligencia emocional y la actitud pedagógica de los docentes de la Universidad Nacio- 
nal Federico Villarreal - 2016 (Tesis doctoral). Universidad Nacional de Educación Enrique Guzmán y Valle, Lima, Perú. Recuperado de http://repositorio.une.edu. pe/handle/UNE/1639

Contreras, M., \& Dextre, G. (2016). Inteligencia emocional y estrés laboral en docentes universitarios de la ciudad de Huancayo (Tesis de licenciatura). Universidad Continental, Huancayo, Perú. https://hdl.handle. net/20.500.12394/2956

Danvila, I., \& Sastre, M. (2010). Inteligencia Emocional: una revisión del concepto y líneas de investigación. Cuader nos de Estudios Empresariales, 20, 107-126. Recuperado de https://revistas.ucm.es/index.php/CESE/article/ view/38963/37595

Fernández, M., Palomero, J., \& Teruel, M. (2009). El desarrollo socioafectivo en la formación inicial de los maestros. Revista Electrónica Interuniversitaria de Formación del Profesorado, 12(1), 33-50. Recuperado de http://www.redalyc.org/pdf/2170/217015332003.pdf

García-Rangel, E., García, A., \& Reyes, J. (2014). Relación maestro alumno y sus implicaciones en el aprendizaje. Ra Ximhai, 10(5), 279-290. https://doi.org/10.35197/ rx.10.03.e1.2014.19.eg

Gallagher, M., Lopez, S., \& Preacher, K. (2009). The Hierarchical Structure of Well-Being. Journal of Personality, 77(4), 1025-1050. https://doi.org/10.1111/j.14676494.2009.00573.x

Golombek, P., \& Doran, M. (2014). Unifying cognition, emotion, and activity in language teacher professional development. Teaching and Teacher Education, 39, 102-111. https://doi.org/10.1016/j.tate.2014.01.002

González-Peiteado, M., Pino-Juste, M., \& Penado-Abilleira, M. (2017). Estudio de la satisfacción percibida por los estudiantes de la UNED con su vida universitaria. RIED. Revista Iberoamericana de Educación a Distancia, 20(1), 243-260. https://doi.org/10.5944/ried.20.1.16377

Hernández, R., Fernández, C., \& Baptista, P. (2014). Metodología de la investigación. México: McGraw-Hill.

Inzunza, B., Ortiz, L., Pérez, C., Torres, G., McColl, P., Meyer, A., ... Bustamante, C. (2015). Estructura Factorial y Confiabilidad del Cuestionario de Satisfacción Académica en Estudiantes de Medicina Chilenos. Revista Iberoamericana de Diagnóstico y Evaluación - e Avaliação Psicológica, 2(40), 73-82. Recuperado de https:// www.redalyc.org/pdf/4596/459645432008.pdf

Jennings, P., \& Greenberg, M. (2009). The Prosocial Classroom: Teacher Social and Emotional Competence in Relation to Student and Classroom Outcomes. Review of Educational Research, 79(1), 491-525. https://doi. org/10.3102/0034654308325693

Keyes, C., Shmotkin, D., \& Ryff, C. (2002). Optimizing well-being: The empirical encounter of two traditions. Journal of Personality and Social Psychology, 82(6), 10071022. http://doi.org/10.1037/0022-3514.82.6.1007

Kline, R. (2011). Principles and practice of structural equation modeling (3 ed.). Nueva York: Guilford Press

Lent, R. (2004). Toward a Unifying Theoretical and Practical Perspective on Well-Being and Psychosocial Adjustment. Journal of Counseling Psychology, 51(4), 482509. http://doi.org/10.1037/0022-0167.51.4.482

Lent, R., Singley, D., Sheu, H., Schmidt, J., \& Schmidt, L. (2007). Relation of Social-Cognitive Factors to Academic Satisfaction in Engineering Students. Journal of Career Assessment, 15(1), 87-97. https://doi. org/10.1177/1069072706294518

Lounsbury, J., Park, S., Sundstrom, E., Williamson, J., \& Pemberton, A. (2004). Personality, Career Satisfaction, and Life Satisfaction: Test of a Directional Model. Journal of Career Assessment, 12(4), 395-406. https://doi. org/10.1177/1069072704266658

Luque-Reca, O., Augusto-Landa, J., \& Pulido-Martos, M. (2016) Emotional intelligence and depressive symptoms in Spanish institutionalized elders: does emotional self-efficacy act as a mediator? PeerJ, 4. https://doi. org/10.7717/peerj.2246

Maidana, A., \& Samudio, M. (2018). Inteligencia emocional de docentes de enfermería según aptitud demostrada en su entorno laboral. Medicina Clínica y Social, 2(2), 72-83.

Mandell, B., \& Pherwani, S. (2003). Relationship between emotional intelligence and transformational leadership style: A gender comparison. Journal of $\mathrm{Bu}$ siness and Psychology, 17(3), 387-404. https://doi.org/10.1023/A:1022816409059

Marzo, M., Iglesias, M., \& Torres, P. (2006). Las competencias profesionales demandadas por las empresas: el caso de los ingenieros. Revista de Educación, 341, 643-661.

Medrano, L., \& Pérez, E. (2010). Adaptación de la escala de satisfacción académica a la población universitaria de Córdoba. Summa Psicológica UST, 7(2), 5-14. https:// doi.org/10.18774/448x.2010.7.117

Merino-Soto, C., Dominguez-Lara, S., \& Fernández-Arata, M. (2017). Validación inicial de una Escala Breve de Satisfacción con los Estudios en estudiantes universitarios de Lima. Educación Médica, 18(1), 74-77. https://doi. org/10.1016/j.edumed.2016.06.016

Monereo, C., \& Pozo, J. (2003). La universidad ante la nueva cultura educativa. Madrid: Síntesis. 
Neri, J., \& Hernández, C. (2019). Los jóvenes universitarios de ingeniería y su percepción sobre las competencias blandas. Revista Iberoamericana para la Investigación y el Desarrollo Educativo, 9(18), 768-791. https:// doi.org/10.23913/ride.v9i18.449

Novoa, C., \& Barra, E. (2015). Influencia del apoyo social percibido y los factores de personalidad en la satisfacción vital de estudiantes universitarios. Terapia psicológica, 33(3), 239-245. http://doi.org/10.4067/ S0718-48082015000300007

Palomera, R., Briones, E., \& Gómez-Linares, A. (2017). Diseño, desarrollo y resultados de un programa de educación socio-emocional para la formación de docentes a nivel de grado y posgrado. Contextos Educativos. Revista de Educación, O(20), 165-182. http://doi.org/10.18172/ con.2988

Palomera, R., Fernández-Berrocal, P., \& Brackett, M. (2008). La inteligencia emocional como una competencia básica en la formación inicial de los docentes: algunas evidencias. Electronic Journal of Research in Educational Psychology, 6(2), 437-454. Recuperado de http://www. redalyc.org/pdf/2931/293121924010.pdf

Peñalva, A., López, J., \& Landa, N. (2012). Competencias emocionales del alumnado de Magisterio: posibles implicaciones profesionales. Revista de Educación, (362), 690-712. https://doi.org/10.4438/1988-592XRE-2013-362-246

Pertegal-Felices, M., Castejón-Costa, J., \& Martínez, M. (2011). Competencias socioemocionales en el desarrollo profesional del maestro. Educación XX1, 14(2), 237-260. https://doi.org/10.5944/educxx1.14.2.253

Ryff, C., \& Keyes, C. (1995). The structure of psychological well-being revisited. Journal of Personality and Social Psychology, 69(4), 719-727. https://doi. org/10.1037/0022-3514.69.4.719

Sánchez, M., Fernández-Berrocal, P., Montañés, J., \& Latorre, J. (2008). ¿Es la inteligencia emocional una cuestión de género? Socialización de las competencias emocionales en hombres y mujeres y sus implicaciones. Elec tronic Journal of Research in Educational Psycholo gy, 6(2), 455-474. Recuperado de http://www.redalyc. org/pdf/2931/293121924011.pdf

Sharma, D. (2017). Impact of age on emotional intelligence and its components. International Journal of Research and Innovation in Social Science (IJRISS), 1(1), 13-20 Recuperado de http://www.ijriss.org/DigitalLibrary/ Vol.1\&Issue1/13-20.pdf

Sisto, F., Muniz, M., Bartholomeu, D., Pasetto, N., Oliveira, A., \& Lopes, W. (2008). Estudo para a construção de uma escala de satisfação acadêmica para universitários. Avaliação Psicológica, 7(1), 45-55. Recuperado de http:// pepsic.bvsalud.org/pdf/avp/v7n1/v7n1a07.pdf

Thomson, M., \& Palermo, C. (2014). Preservice teachers' understanding of their professional goals: Case studies from three different typologies. Teaching and Teacher Education, 44, 56-68. https://doi.org/10.1016/j. tate.2014.08.002

Villa-Peralta, A. (2017). La formación educativa del ingeniero y la compleja realidad del mundo contemporáneo. Aibi Revista de Investigación, Administración e Ingeniería, 5(2), 9-15. https://doi.org/10.15649/2346030X.435

Zepeda-Hurtado, M., Cardoso-Espinosa, E., \& Rey-Benguría, C. (2019). El desarrollo de habilidades blandas en la formación de ingenieros. Científica, 23(1), 61-67. Recuperado de http://www.redalyc.org/articulo. oa?id=61458265007

RIDU / Revista Digital de Investigación en Docencia Universitaria / e-ISSN: 2223-2516

(C) Los autores. Este artículo es publicado por la Revista Digital de Investigación en Docencia Universitaria del Área de Institutional Research and Effectiveness de la Dirección de Aseguramiento de la Calidad, Universidad Peruana de Ciencias Aplicadas. Este es un artículo de acceso abierto, distribuido bajo los términos de la LicenciaCreativeCommons Atribución-CompartirIgual 4.0 Internacional.(http://creativecommons.org/licenses/by-sa/4.0/), que permite el uso no comercial, distribución y reproducción en cualquier medio, siempre que la obra original sea debidamente citada. 